\title{
DECOMPOSITIONS OF CONTINUA OVER THE HYPERBOLIC PLANE
}

\author{
JAMES T. ROGERS, JR.
}

Dedicated to F. Burton Jones, who first studied decompositions of homogeneous continua

ABSTRACT. The following theorem is proved.

THEOREM. Let $X$ be a homogeneous continuum such that $H^{1}(X) \neq 0$. If $\mathscr{G}$ is the collection of maximal terminal proper subcontinua of $X$, then

(1) The collection $\mathscr{G}$ is a monotone, continuous, terminal decomposition of $X$,

(2) The nondegenerate elements of $\mathscr{G}$ are mutually homeomorphic, indecomposable, cell-like, terminal, homogeneous continua of the same dimension as $X$,

(3) The quotient space is a homogeneous continuum, and

(4) The quotient space does not contain any proper, nondegenerate, terminal subcontinuum.

This theorem is related to the Jones' Aposyndetic Decomposition Theorem. The proof involves the hyperbolic plane and a subset of the circle at $\infty$, called the set of ends of a component of the universal cover of $X$.

F. Burton Jones [11] has proved a marvelous theorem for the study of decomposable, homogeneous continua. Called the Aposyndetic Decomposition Theorem, it is usually stated like this:

THEOREM 1. Let $M$ be a homogeneous continuum such that $M$ is decomposable. If $\mathscr{G}=\{L(x): x \in M\}$, then

(1) The collection $\mathscr{G}$ is a monotone, continuous, terminal decomposition of $M$,

(2) The nondegenerate elements of the decomposition are mutually homeomorphic, cell-like, indecomposable, homogeneous continua of the same dimension as $M$,

(3) The quotient space is a homogeneous continuum, and

(4) The quotient space is an aposyndetic continuum.

This theorem gives a good strategy for studying decomposable, homogeneous continua: Understand the aposyndetic ones, understand the cell-like, indecomposable, homogeneous continua, and put them together to understand decomposable, homogeneous continua.

A similar theorem for indecomposable, homogeneous continua has eluded us so far, even though we've sensed it lurking for twenty years or so [13, p. 49]. In this

Received by the editors June 23, 1986 and, in revised form, August 13, 1987. Presented in the Special Session on Geometric Topology, American Mathematical Society Southeastern Regional Meeting, Charlotte, N.C., 1986.

1980 Mathematics Subject Classification (1985 Revision). Primary 54F20; Secondary 54F50.

Key words and phrases. Continuum, curve, homogeneous, Effros property, aposyndetic decomposition, terminal subcontinuum, hyperbolic plane.

This research was partially supported by a grant from the National Science Foundation. 
paper, we prove a theorem that is just such an analogue; in fact it is a natural extension of the aposyndetic decomposition. Called the Terminal Decomposition Theorem, it goes like this:

THEOREM 2. Let $X$ be a homogeneous continuum such that $H^{1}(X) \neq 0$. If $\mathscr{G}$ is the collection of maximal terminal proper subcontinua of $X$, then

(1) The collection $\mathscr{G}$ is a monotone, continuous, terminal decomposition of $X$,

(2) The nondegenerate elements of $\mathscr{G}$ are mutually homeomorphic, indecomposable, cell-like, terminal, homogeneous continua of the same dimension as $X$,

(3) The quotient space is a homogeneous continuum, and

(4) The quotient space does not contain any proper, nondegenerate terminal subcontinuum.

Hence we have a good strategy for understanding cyclic, homogeneous continua: Understand the ones with no proper, nondegenerate, terminal subcontinuum, understand the cell-like, indecomposable, homogeneous continua, and put them together to understand cyclic, homogeneous continua.

There are two reasons why this terminal decomposition is bound to the aposyndetic decomposition. One is that a homogeneous, decomposable continuum is aposyndetic if and only if it contains no proper, nondegenerate, terminal subcontinuum. Hence condition (4) of Theorem 1 can be replaced by condition (4) of Theorem 2. Furthermore, if $X$ is a decomposable continuum, then the terminal decomposition agrees with the aposyndetic decomposition, since it is explicit in Jones' proof that the $L(x)$ 's are maximal proper terminal subcontinua. Thus we can state both theorems in one.

THEOREM 3. Let $X$ be a homogeneous continuum such that either $X$ is decomposable or $H^{1}(X) \neq 0$. If $\mathscr{G}$ is the collection of maximal terminal proper subcontinua of $X$, then conditions (1)-(4) of Theorem 2 are true.

An outline of the proof of Theorem 2 reveals the second reason that these two theorems are bound together. Let $F$ be a closed, hyperbolic surface and $Q$ the Hilbert cube. Embed $X$ essentially in $F \times Q$. Let $H$ be the hyperbolic plane, and let $H \times Q$ be the universal covering space of $F \times Q$. Let $K$ be a component of the preimage $\tilde{X}$ of $X$ in $H \times Q$. Compactify $K$ by a certain subset of the circle at $\infty$ to get a continuum $Y$.

The continuum $Y$ is aposyndetic at each point of the circle at $\infty$, and $K$ is a homogeneous subset of $Y$. This is just enough aposyndesis and just enough homogeneity to do a modified aposyndetic decomposition of $Y$. Push a typical decomposition element down to $X$ by the covering map, and show it to be a maximal proper terminal subcontinuum of $X$. This decomposition element, pushed down from $Y$, generates the decomposition of $X$. Hence aposyndetic decompositions are essential in obtaining terminal decompositions.

Here is another important aspect of this study. Suppose the decomposition of Theorem 2 has been done; this means that we are considering a homogeneous continuum $X$ such that $H^{1}(X) \neq 0$ and such that $X$ contains no nondegenerate, terminal, proper subcontinuum. When we construct the continuum $Y$, we find that $Y$ is aposyndetic. Since the covering map takes the dense set $K$ of $Y$ into $X$, we can obtain new information about $X$ by studying $Y$, an aposyndetic continuum with lots of homogeneity. 
Specifically, if $X$ is already aposyndetic, then the decomposition itself gives us no new information. The continuum $Y$, however, may give us lots of information; for instance $Y$ might be locally connected.

The Terminal Decomposition Theorem is actually proved under the apparently weaker hypothesis that $X$ admits an essential map into a graph (recall that $H^{1}(X)$ $\neq 0$ if and only if $X$ admits an essential map into $S^{1}$ ). In fact, the graph used is the figure eight. This is not done for the purposes of shape theory, since the author [28] has shown that, for homogeneous curves, trivial cohomology implies trivial shape.

The purpose of using graphs is that this whole process may be considered to be "unwinding $X$ over the hyperbolic plane," and a graph of higher genus may yield "more unwinding." In particular, for the Minc-Rogers examples [21] of homogeneous curves, which involve retracting the Menger universal curve onto a wedge $G$ of circles, it is clearly desirable to let the graph be $G$ and to modify the construction in this paper by using a closed hyperbolic surface $F$ of genus sufficiently high that $G$ can be embedded in $F$ so that every circle in $G$ represents a generator of $\pi_{1}(F)$.

In addition to Jones, earlier work on decompositions of homogeneous continua has been done by Bellamy and Lum [1], Hagopian [7, 8, 9], Maćkowiak and Tymchatyn $[\mathbf{1 9}]$, Lewis $[\mathbf{1 7}, \mathbf{1 8}]$ and the author $[\mathbf{2 2}, \mathbf{2 3}, \mathbf{2 6}]$.

Here is the organization of the paper. $\S 2$ is devoted entirely to proving the essential Theorem 2.5. The reader may wish to begin at $\S 3$ and read $\S 2$ later. In $\S 3$, the covering space is constructed. $\S 4$ is the heart of the paper; it includes the proof of the Terminal Decomposition Theorem.

In $\S 5$, the study of the continuum $Y$ associated with $X$ is initiated. $\S 6$ contains simple proofs of two known theorems; it can be read without referring to the other sections.

A continuum is a compact, connected, nonvoid metric space. A curve is a onedimensional continuum. A space is homogeneous if its homeomorphism group acts transitively on it.

A metric space $X$ has the Effros property if given $\varepsilon>0$, there exists $\delta>0$ such that whenever $y$ and $z$ are points of $X$ satisfying $d(y, z)<\delta$, there exists a homeomorphism $h: X \rightarrow X$ such that $h(y)=z$ and $d(x, h(x))<\varepsilon$, for all $x$ in $X$. Such a $\delta$ is called an Effros $\delta$ for $\varepsilon$. Effros [5] has shown that each homogeneous continuum has the Effros property.

A continuum is decomposable if it is the union of two of its proper subcontinua. Otherwise it is indecomposable. A continuum is hereditarily indecomposable if it contains no decomposable subcontinuum.

A continuum is cell-like (or of trivial shape) if each map of it into a polyhedron is inessential. Hence a curve $X$ is cell-like if each map of it into a graph is inessential. It is known [16 or 20] that a curve $X$ has an essential map into a graph if and only if $X$ has an essential map into a "figure eight." Cell-like curves are usually called tree-like continua.

2. Aposyndetic decompositions. The goal of this section is to prove Theorem 2.5. This generalization of the Jones' Aposyndetic Decomposition Theorem will be used in the fourth section.

Our strategy of proof is to give a little different proof of the Jones' Theorem than is currently in the literature and to do it in such a way that the proof of the 
generalization is straightforward. The ideas of Jones are used extensively, of course, and the improvements of the author [26 and 30] are included.

Let $x$ and $y$ be points of the continuum $M$. If $M$ contains an open set $G$ and a continuum $H$ such that $x \in G \subset H \subset M-\{y\}$, then $M$ is said to be aposyndetic at $x$ with respect to $y$. If $M$ is aposyndetic at $x$ with respect to every other point of $M$, then $M$ is aposyndetic at $x$. If $M$ is aposyndetic at each of its points with respect to every other point, then $M$ is said to be aposyndetic.

The set $L(x)$ has as members the point $x$ together with all points $z$ of $M$ such that $M$ is not aposyndetic at $z$ with respect to $x$. Each set $L(x)$ is a continuum. If $M$ is indecomposable, then $L(x)=M$, for all $x$ in $M$.

A partition of a space $M$ is a collection of disjoint sets whose union is $M$. The heart of Jones' Theorem is to partition a homogeneous continuum using aposyndesis.

THEOREM 2.1. Let $M$ be a homogeneous continuum. If $\mathscr{G}=\{L(x): x \in M\}$, then $\mathscr{G}$ is a partition of $M$.

ProOF. Let $x$ belong to $M$. We must show that if $y$ belongs to $L(x)$, then $L(y)=L(x)$.

First we show $L(y) \subset L(x)$. For each point $p$ not in $L(x)$, there exists a continuum $W \subset M-x$ containing $p$ in its interior. If $p$ also belongs to $L(y)$, then $y$ belongs to $W$. Choose some small homeomorphisms of $M$ so that the images of $W$ all intersect the interior of $W$ and so that the closure of the union of these images covers a small neighborhood of $y$ and misses $x$. This implies the contradiction that $y$ does not belong to $L(x)$. Hence $L(y) \subset L(x)$.

To show $L(x) \subset L(y)$, we use an argument of Bellamy and Lum [1, Lemma 5]. We find one point $x_{0}$ for which this is true; the homogeneity of $M$ then implies it is true for every point of $M$.

Partially order $\mathscr{G}$ with respect to inclusion. Choose a maximal chain $R$. Since each $L(x)$ is a nonempty continuum, there exists a point $x_{0}$ in $R$. Since $R$ is maximal, $L\left(x_{0}\right)$ is the minimal element of $R$. Thus Zorn's Lemma implies $L\left(x_{0}\right) \subset$ $L(y)$, for each $y$ in $L\left(x_{0}\right)$. This completes the proof of the theorem.

Let $G$ be a partition of a continuum $M$. To say that a set $H$ of homeomorphisms of $M$ respects $G$ means that either $h\left(G_{1}\right)=G_{2}$ or $h\left(G_{1}\right) \cap G_{2}=\varnothing$, for all $G_{1}$ and $G_{2}$ in $\mathscr{G}$ and all $h$ in $H$.

A partition $\mathscr{G}$ of a continuum $M$ is a continuous decomposition of $M$ if the quotient space is a continuum and the quotient function of $M$ onto the quotient space is an open map.

The next theorem is the Decomposition Theorem for Homogeneous Continua [23].

THEOREM 2.2. Let $\mathscr{G}$ be a partition into proper subcontinua of the homogeneous continuum $M$. If the homeomorphism group of $M$ respects $\mathscr{G}$, then

(1) The partition $\mathscr{G}$ is a continuous decomposition of $M$,

(2) The elements of $\mathscr{G}$ are mutually homeomorphic, homogeneous continua, and

(3) The quotient space is a homogeneous continuum.

A subcontinuum $Z$ of the continuum $X$ is said to be terminal if each subcontinuum $Y$ of $X$ that intersects $Z$ satisfies either $Y \subset Z$ or $Z \subset Y$. A decomposition 
of $X$ into continua is said to be terminal if each element of the decomposition is a terminal subcontinuum of $X$.

The author $[\mathbf{2 6}, \mathbf{3 0}]$ has proved the following theorem about terminal, continuous decompositions.

THEOREM 2.3. If $\mathscr{G}$ is a terminal, continuous decomposition of the homogeneous continuum $M$, if the elements of $\mathscr{G}$ are proper, nondegenerate subcontinua, and if the homeomorphism group of $M$ respects $\mathscr{G}$, then the elements of $\mathscr{G}$ are cell-like, indecomposable continua of the same dimension as $M$.

We now have all the ingredients to prove the important Jones' Aposyndetic Decomposition Theorem.

THEOREM 2.4. Let $M$ be a homogeneous, decomposable continuum that is not aposyndetic. If $\mathscr{G}=\{L(x): x \in M\}$, then

(1) The collection $\mathscr{G}$ is a monotone, continuous, terminal decomposition of $M$,

(2) The elements of the decomposition are mutually homeomorphic, cell-like, indecomposable, homogeneous continua of the same dimension as $M$,

(3) The quotient space is a homogeneous continuum, and

(4) The quotient space is an aposyndetic continuum.

ProOF. Since $M$ is decomposable, $M$ is aposyndetic at some point with respect to some other point. Thus the elements of $\mathscr{G}$ are proper nondegenerate subcontinua of $M$. By Theorem 2.1, $\mathscr{G}$ is a partition of $M$. Since $\mathscr{G}$ is defined topologically, the homeomorphism group $H(M)$ respects $G$, and all the conclusions of Theorem 2.2 hold.

That the decomposition $\mathscr{G}$ is terminal may be shown by the argument in paragraph two of the proof of Theorem 2.1. The elements of this terminal, continuous decomposition are cell-like and of the same dimension as $M$, by Theorem 2.3.

Furthermore, if $G_{1}$ and $G_{2}$ are different elements of $\mathscr{G}$, and $K$ is a subcontinuum of $M$ containing a point of $G_{1}$ in its interior and missing a point of $G_{2}$, then $K$ contains no point of $G_{2}$ and all points of $G_{1}$. Hence the quotient space is aposyndetic. This completes the proof.

Finally we are ready to prove an alternate version of the Aposyndetic Decomposition Theorem. The awkward collection of hypotheses in this theorem is precisely the situation encountered in $\S 4$.

It may help the reader to picture $Y$ as an arc of pseudo-arcs with the two end pseudo-arcs shrunk to points $z_{1}$ and $z_{2}$. In this case, picture $E$ as $\left\{z_{1}, z_{2}\right\}$, and $K=Y-E$.

THEOREM 2.5. Let the continuum $Y$ be the union of two disjoint, nonempty sets $K$ and $E$ with the following properties:

(1) $Y$ is aposyndetic at each point of $E$,

(2) $Y$ is not aposyndetic at any point of $K$,

(3) $Y$ is aposyndetic at each point of $K$ with respect to each point of $E$,

(4) $K$ has a metric $d$ such that $(K, d)$ has the Effros property,

(5) Each bounded homeomorphism $h$ of $(K, d)$ can be extended to a homeomorphism $\hat{h}$ of $Y$, by defining $\hat{h}(z)=z$, for each $z$ in $E$,

(6) $K$ is connected and open, and 
(7) $\operatorname{dim} E=0$.

If $\mathscr{G}=\{L(x): x \in Y\}$, then $\mathscr{G}$ is a continuous, terminal decomposition of $Y$ with the following properties:

(8) The degenerate elements of $\mathscr{G}$ are precisely the points of $E$,

(9) The nondegenerate elements of $\mathscr{G}$ are mutually homeomorphic, cell-like, homogeneous, indecomposable continua of the same dimension as $Y$,

(10) The quotient space of $Y$ is aposyndetic, and

(11) The quotient space of $K$ is homogeneous.

PROOF. Since $Y$ is aposyndetic at every point with respect to at least one other point, the elements of $\mathscr{G}$ are proper subcontinua of $M$. Moreover, if $x$ belongs to $K$, then $L(x)$ is contained in $K$, since $Y$ is aposyndetic at each point of $E$. Furthermore, if $x$ belongs to $E$, then $L(x)$ is the singleton set $\{x\}$, since $Y$ is aposyndetic at every other point of $Y$ with respect to $x$.

Thus the elements of $\mathscr{G}$ are the points of $E$ and some nondegenerate subcontinua of $K$. An argument similar to the proof of Theorem 2.1 shows that $\mathscr{G}$ is a partition of $Y$. Since $\mathscr{G}$ is defined topologically, the homeomorphism group of $Y$ respects $\mathscr{G}$.

We cannot use the Decomposition Theorem directly, but we can modify the proof to apply to this situation. It is easy to use the Effros property for $K$ to prove the partition is a continuous decomposition at every element of $\mathscr{G}$ contained in $K$. Since the elements of $\mathscr{G}$ contained in $E$ are points and $\operatorname{dim} E=0$, it follows that $\mathscr{G}$ is a continuous decomposition.

Each degenerate element of $\mathscr{G}$ is obviously a terminal subcontinuum of $Y$. A proof that the nondegenerate elements of $\mathscr{G}$ are terminal subcontinua may be fashioned after the second paragraph of the proof of Theorem 2.1.

Clearly the nondegenerate elements of $\mathscr{G}$ are mutually homeomorphic, homogeneous continua. Since $\mathscr{G}$ is a terminal, continuous decomposition, the nondegenerate elements of $\mathscr{G}$ are cell-like and of the same dimension as $Y$, by a proof similar to that of Theorem 2.3. That one, and hence each nondegenerate element, is an indecomposable continuum that can be deduced from [19, Theorem 6.13].

The proofs that the quotient space of $Y$ is an aposyndetic continuum and that the quotient space of $K$ is a homogeneous space are straightforward.

3. The ends of the universal covers of certain continua. Let $H$ be the interior of the closed unit disk $D$ in the Euclidean plane, and let $S^{1}$ be its boundary.

A geodesic in $H$ is the intersection of $H$ and a circle $C$ in the Euclidean plane that intersects $S^{\mathbf{1}}$ orthogonally (straight lines through the origin are considered circles centered at $\infty$ ).

A reflection in the geodesic $C \cap H$ is Euclidean inversion in the circle $C$. An isometry of $H$ is a product of reflections in geodesics.

The set $H$, with this set of isometries, is a surface of constant negative curvature isometric to hyperbolic space. This model of hyperbolic space is called the Poincaré disc. The boundary $S^{1}$ of $H$, which is not in $H$, is called the circle at $\infty$.

Let $F$ be a closed, orientable surface of genus two. The universal cover of $F$ can be chosen to be $H$, and the group of deck transformations to be a subgroup of the orientation-preserving isometries of $H$. Each of these deck transformations (except the identity map) is a hyperbolic isometry. The pertinent property for us is that a hyperbolic isometry, when extended to the circle at $\infty$, has exactly two fixed 


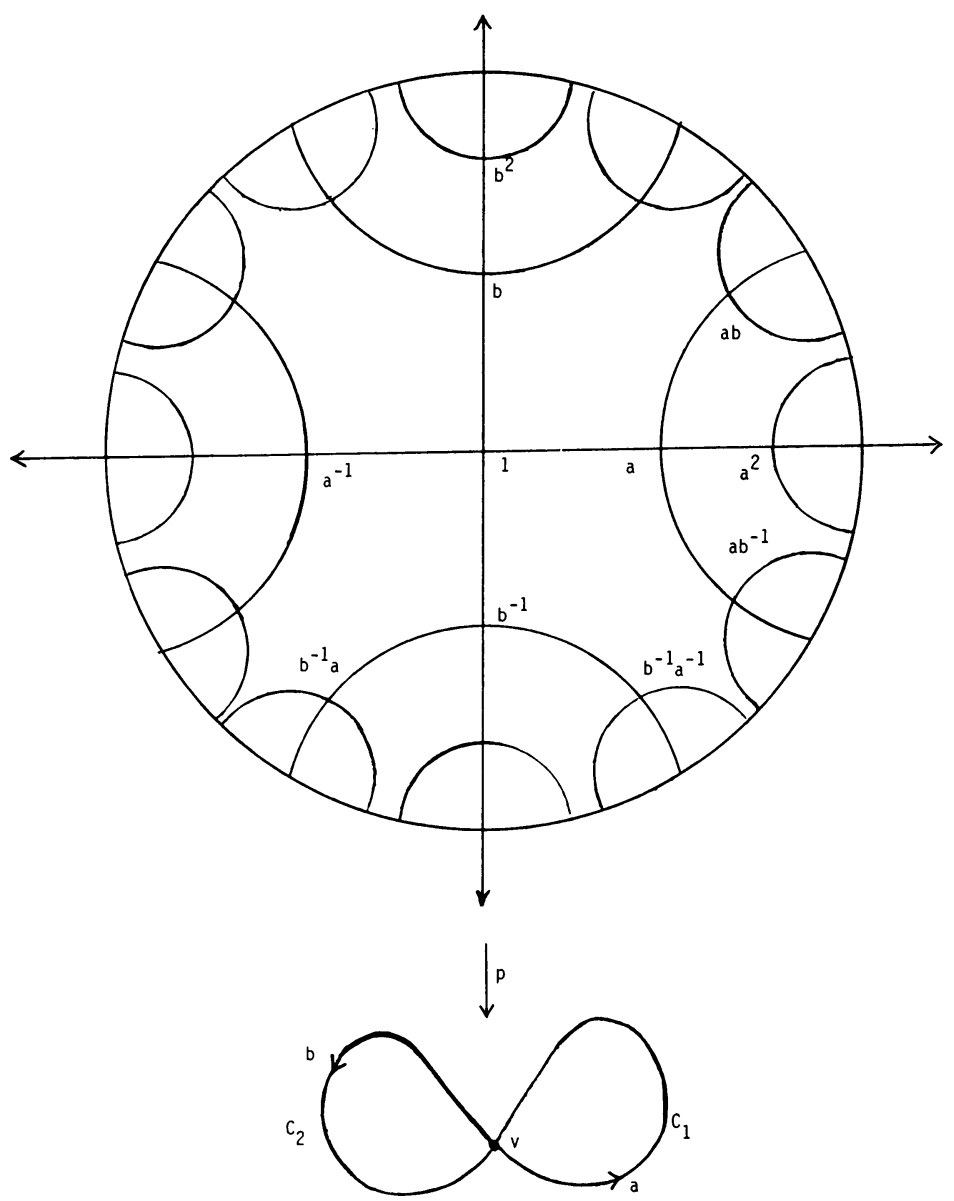

Figure 1. The universal cover $\tilde{W}$ of $W$ in the hyperbolic plane $H$ and labeled as the Cayley graph of $\pi_{1}(W, v)$

points in $S^{1}$ and none in $H$. One fixed point is attracting, and one is repelling. In particular, a hyperbolic isometry does not leave any compact subspace of $H$ fixed (setwise).

Let $p: H \rightarrow F$ be the universal covering map. We may assume the map $p$ is a local isometry.

A geodesic in $F$ is the image under $p$ of a geodesic in $H$. A simple closed geodesic is a geodesic that is also a simple closed curve.

Assume the universal covering space $(H, p, F)$ of $F$ is constructed with the following properties. The geodesic $H \cap x$-axis maps to a simple closed geodesic $C_{1}$. The geodesic $H \cap y$-axis maps to a simple closed geodesic $C_{2}$. Furthermore, the union of $C_{1}$ and $C_{2}$ is a figure eight $W$, and the intersection of $C_{1}$ and $C_{2}$ is a single point $v$.

Let $\tilde{W}=p^{-1}(W)$; hence $\tilde{W}$ is the universal cover of a figure eight, the "infinite snowflake" pictured in Figure 1. The set $\mathrm{Cl}(\tilde{W}) \cap S^{1}$ is a Cantor set; call it $Z$. 
Let $Q$ be a Hilbert cube, and let $p \times 1: H \times Q \rightarrow F \times Q$ be the universal covering space of $F \times Q$. Let $X$ be a continuum embedded essentially in $W \times Q$ (this means that the inclusion map is essential; note that this eliminates some continua from consideration). Let $f: X \rightarrow W$ be the projection map.

Let $\tilde{X}=(p \times 1)^{-1}(X)$, and let $\tilde{f}: \tilde{X} \rightarrow \tilde{W}$ be the projection map. Let $K$ be a component of $\tilde{X}$.

Define $E(K)=\left\{z \in S^{1}: z\right.$ is a (Euclidean) limit point of $\left.\tilde{f}(K)\right\}$. Call $E(K)$ the set of ends of $K$.

In [28], the following theorems were established.

THEOREM 3.1. The set $K$ is unbounded, and hence $E(K)$ is a nonempty, closed subset of $S^{1}$.

THEOREM 3.2. If $X$ is homogeneous, then $\tilde{X}$, and hence $K$, have the Effros property. In particular, there exists an $\varepsilon>0$ such that each $\varepsilon$-homeomorphism of $X$ lifts to an $\varepsilon$-homeomorphism of $\tilde{X}$.

THEOREM 3.3. If $X$ is homogeneous, then $E(K)$ is either a two-point set or a Cantor set. Furthermore, $E(K)$ contains a dense subset each point of which is a fixed point of a hyperbolic isometry $\phi$ such that $\phi \times 1$ is a deck transformation.

If $a$ and $b$ denote generators of $\pi_{1}(W, v)$, then the vertices of $\tilde{W}$ may be labeled with elements of the group $\pi_{1}(W, v)$ as indicated in Figure 1 . In this sense, $\tilde{W}$ is the Cayley graph of $\pi_{1}(W, v)$.

A point $w$ in $\tilde{W}$ and a point $z$ in $Z$ determine a unique arc $[w, z]$ in $\operatorname{Cl}(\tilde{W})$ from $w$ to $z$. The arc $[w, z]$ determines an infinite word in the letters $a$ and $b$. For example, if $w=(0,0)$ and $z=(1,0)$, then the word is $a a a \cdots$. If $w=(0,0)$ and $z=(-1,0)$, then the word is $a^{-1} a^{-1} a^{-1} \ldots$.

A vertex $q$ on the arc $[w, z]$ is two letters closer to $z$ than the vertex $p$ on $[w, z]$ if $q$ separates $p$ from $z$ in $[w, z]$ and $[p, q]$ contains a vertex of $\tilde{W}$.

Compactify $H \times Q$ with $S^{1} \times Q$. Shrink each set of the form $\{z\} \times Q$, where $z$ is a point of $S^{1}$, to a point to obtain another compactification of $H \times Q$. This time the remainder is $S^{1}$.

Let $\pi:(H \times Q) \cup S^{1} \rightarrow H \cup S^{1}$ be the map of this latter compactification onto the disk $D$ obtained by naturally extending the projection map. Note that the restriction of $\pi$ to $\tilde{X}$ is just $\tilde{f}$.

Let $Y=\mathrm{Cl}(K)$ in $(H \times Q) \cup S^{1}$; then $Y-K=E(K)$. In the next section, we shall take an aposyndetic decomposition of $Y$.

4. Aposyndetic decompositions over the hyperbolic plane. Assume $X$ is a homogeneous continuum that admits an essential map into the figure eight $W$. Hence $X$ can be embedded essentially in $W \times Q$, and all of the theorems of the preceding section apply to $X$.

Assume each of the simple closed geodesics $C_{1}$ and $C_{2}$ have length greater than one. Let $2 \delta$ be an Effros $\delta$ for $\varepsilon=1 / 2$.

A space $S$ is connected im kleinen at a point $x$ if for every neighborhood $U$ of $x$, there is a connected subset $A$ of $U$ that contains a neighborhood of $x$.

THEOREM 4.1. If $X$ is homogeneous, then the continuum $Y$ is connected im kleinen at each point of $E(K)$. 

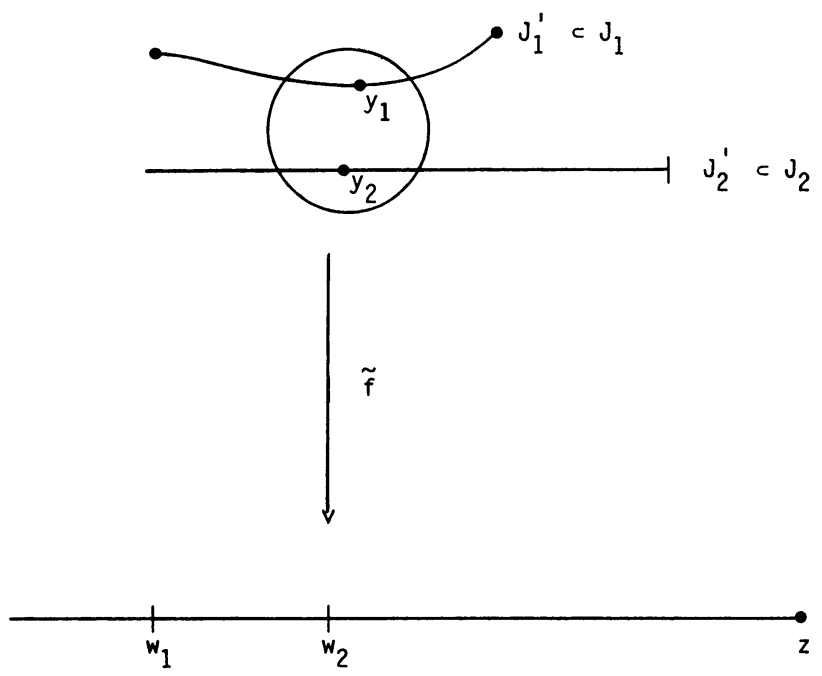

FIGURE 2

PROOF. Let $z$ belong to $E(K)$. It suffices to show that $z$ is an interior point of the component $J$ of $z$ in $Y-\pi^{-1}\left(w_{1}\right)$, where $w_{1}$ is an arbitrary point of $\pi(K)$. This is because we can use a deck transformation $\phi$ to move $w_{1}$ as close to $z$ as we like, by Theorem 3.3.

If $z$ is not an interior point of $J$, there exists a sequence $\left\{J_{n}\right\}$ of components of $Y-\pi^{-1}\left(w_{1}\right)$ such that, for all $n$,

(1) $J \cap J_{n}=\phi$, and

(2) $\tilde{f}\left(J_{n+1}\right)$ contains a vertex two letters closer to $z$ than any vertex of $\tilde{f}\left(J_{n}\right)$.

Let $w_{2}$ be a vertex on $\left[w_{1}, z\right]$ two letters closer to $z$ than $w_{1}$. For each $n$, let $J_{n}^{\prime}$ denote a component of $J_{n}-\pi^{-1}\left(w_{2}\right)$ such that $\tilde{f}\left(J_{n+1}^{\prime}\right)$ contains a vertex two letters closer to $z$ than any vertex of $\tilde{f}\left(J_{n}^{\prime}\right)$.

Cover $\left\{w_{2}\right\} \times Q$ with a finite number of $\delta$-balls, where $2 \delta$ is an Effros $\delta$ for $\varepsilon=1 / 2$. We will show no $\delta$-ball can intersect more than one of the sets $J_{n}^{\prime}$. This contradiction will prove the theorem.

Suppose some $\delta$-ball intersects more than one $J_{n}^{\prime}$. Without loss of generality, assume the $\delta$-ball $B$ intersects both $J_{1}^{\prime}$ and $J_{2}^{\prime}$. Let $y_{1}$ be a point of $B \cap J_{1}^{\prime}$, and $y_{2}$ a point of $B \cap J_{2}^{\prime}$ (see Figure 2). Let $h: K \rightarrow K$ be an $\varepsilon$-homeomorphism such that $h\left(y_{2}\right)=y_{1}$. Since $J_{2}^{\prime}$ is connected and $h$ is an $\varepsilon$-homeomorphism, we can conclude that $h\left(J_{2}^{\prime}\right) \subset J_{1}$ and that $h\left(J_{2}^{\prime}\right) \not \subset J_{1}$.

This contradiction completes the proof of the theorem.

COROLlaRY 4.2. The continuum $Y$ is aposyndetic at each point of $E(K)$.

Since $Y=K \cup E(K)$, it is clear that the following theorem is an immediate consequence of Theorem 2.5 .

THEOREM 4.3. The collection $\{L(x): x \in Y\}$ is a continuous, terminal decomposition of $Y$ with the following properties:

(1) The quotient space of $Y$ is aposyndetic.

(2) $L(x)$ is degenerate if $x$ belongs to the circle at $\infty$. 
(3) The nondegenerate elements of the decomposition are mutually homeomorphic, cell-like, indecomposable, homogeneous continua of the same dimension as $Y$.

(4) The quotient space of $K$ is homogeneous.

We need three more theorems before we are ready to define the decomposition $\mathscr{G}$ of $X$.

THEOREM 4.4. If $x$ belongs to $K$ and $L(x)$ is the decomposition element containing $x$, then the restriction of the covering map $p \times 1$ to $L(x)$ is one-to-one.

PROOF. Let $x_{1}$ and $x_{2}$ be distinct points of $L(x)$ with the property that $(p \times 1)\left(x_{1}\right)=(p \times 1)\left(x_{2}\right)$. Let $\phi: H \times Q \rightarrow H \times Q$ be the deck transformation satisfying $\phi\left(x_{1}\right)=x_{2}$. Since $\phi$ does not fix (setwise) any compact set, it follows that $\phi(L(x)) \cap(Y-L(x)) \neq \varnothing$ and $L(x) \cap(Y-\varnothing(L(x))) \neq \varnothing$. Since $\phi(L(x)) \cap L(x) \neq \varnothing$, this contradicts the fact that the decomposition is terminal.

THEOREM 4.5. If $x_{1}$ and $x_{2}$ are points of $\tilde{X}$ such that $(p \times 1)\left(x_{1}\right)=(p \times 1)\left(x_{2}\right)$, then $L\left(x_{1}\right)$ is homeomorphic to $L\left(x_{2}\right)$, and $(p \times 1)\left(L\left(x_{1}\right)\right)=(p \times 1)\left(L\left(x_{2}\right)\right)$.

PROOF. Let $\phi: H \times Q \rightarrow H \times Q$ be the deck transformation satisfying $\phi\left(x_{1}\right)=x_{2}$. Since the aposyndetic decomposition is defined topologically, it follows that

$$
\phi\left(L\left(x_{1}\right)\right)=L\left(\phi\left(x_{1}\right)\right)=L\left(x_{2}\right) .
$$

Hence $L\left(x_{1}\right)$ is homeomorphic to $L\left(x_{2}\right)$, and $(p \times 1)\left(L\left(x_{1}\right)\right)=(p \times 1)\left(L\left(x_{2}\right)\right)$.

THEOREM 4.6. The continuum $(p \times 1)(L(x))$ is a maximal terminal proper, cell-like subcontinuum of $X$.

PROOF. Since $L(x)$ is cell-like, it follows from Theorem 4.4 that $(p \times 1)(L(x))$ is cell-like. Hence $(p \times 1)(L(x))$ is a proper subcontinuum of $X$.

First we show $(p \times 1)(L(x))$ is a terminal subcontinua of $X$. It is easy to show that $p \times 1$ being one-to-one on $L(x)$ implies that $p \times 1$ is one-to-one on some (open) neighborhood $U$ of $L(x)$.

If $M$ is a subcontinuum of $X$ containing a point of both $(p \times 1)(L(x))$ and its complement, then we can assume, by taking a subcontinuum if necessary, that $M \subset(p \times 1)(U)$. Hence $M$ has a lift $\tilde{M}$ that contains points of both $L(x)$ and its complement. Since $L(x)$ is terminal, $\tilde{M}$ contains $L(x)$ and hence $M$ contains $(p \times 1)(L(x))$. Hence $(p \times 1)(L(x))$ is a terminal subcontinuum of $X$.

Second, we show that if $T$ is a proper, terminal, cell-like subcontinuum of $X$ containing $(p \times 1)(L(x))$, then $T=(p \times 1)(L(x))$. Since $T$ is cell-like, the inclusion map $i: T \rightarrow W \times Q$ lifts to an injection $\tilde{i}: T \rightarrow \tilde{W} \times Q$ such that $\tilde{i}(T)$ meets $L(x)$. Let $\tilde{T}$ denote $\tilde{i}(T)$.

Since $p \times 1$ is one-to-one on $\tilde{T}$, there is a neighborhood $U$ of $\tilde{T}$ such that $p \times 1$ is one-to-one on $U$. Since $L(x)$ is a maximal terminal proper subcontinuum of $Y, \tilde{T}$ is not terminal in $Y$ or $\tilde{T}=L(x)$. Let $M$ be a proper subcontinuum of $Y$ containing a point of $\tilde{T}$ and a point of its complement. Again we can assume $M \subset U$. Hence $(p \times 1)(M)$ is a continuum containing a point of $T$ and a point of its complement. Since $T$ is terminal, $(p \times 1)(M)$ contains $T$. Hence $M$ contains $\tilde{T}$ and so $\tilde{T}$ is terminal. Hence $\tilde{T}=L(x)$, and $T=(p \times 1)(L(x))$. This completes the proof of the theorem. 
We are ready to state and prove the main theorem of the paper.

THEOREM 4.7. Let $X$ be a homogeneous continuum that admits an essential map into a graph $D$. If $\mathscr{G}$ is the collection of maximal terminal proper subcontinua of $X$, then

(1) The collection $\mathscr{G}$ is a monotone, continuous, terminal decomposition of $X$.

(2) The nondegenerate elements of $\mathscr{G}$ are mutually homeomorphic, indecomposable, cell-like, terminal, homogeneous continua of the same dimension as $X$.

(3) The quotient space is a homogeneous continuum.

(4) The quotient space does not contain any proper, nondegenerate terminal subcontinuum.

(5) If $X$ is decomposable, then the quotient space is an aposyndetic continuum; in fact, the decomposition is the Jones' decomposition, and

(6) The quotient space admits an essential map into the graph $D$.

Proof. The only difficult part of this proof is to show that $\mathscr{G}$ exists, i.e., that $X$ contains a maximal terminal proper subcontinuum.

Let $\mathscr{G}$ be the collection $\{(p \times 1)(L(x)): x \in \tilde{X}\}$. This is the collection of maximal terminal proper, cell-like subcontinua.

Since $\mathscr{G}$ is defined topologically, the homeomorphism group of $X$ respects $\mathscr{G}$. Furthermore, since the elements of $\mathscr{G}$ are maximal terminal proper, cell-like subcontinua of $X$, it follows that $\mathscr{G}$ is a partition of $X$ into proper subcontinua.

Therefore, Theorem 2.2 implies that conditions (1) and (3) are satisfied. Furthermore, Theorem 2.2 and Theorem 2.3 imply that condition (2) is satisfied. Conditions (4) and (5) are easily seen to hold.

Condition (6) follows from a result [6] of Grispolakis and Tymchatyn. If the graph $D$ is $S^{1}$, this is just the Vietoris-Begle Theorem.

Finally, we show that if $T$ is a proper, terminal subcontinuum of $X$ containing $(p \times 1)(L(x))$, then $T=(p \times 1)(L(x))$. If not, then it follows from the arguments of the preceding theorem that the inclusion map $i: T \subset X$ is an essential map. We may assume that $T$ is minimal with respect to the properties of (1) containing $(p \times 1)(L(x)),(2)$ being terminal, and (3) being embedded essentially in $W \times Q$.

Being embedded essentially in $W \times Q$ is a property preserved by $\varepsilon$-homeomorphisms, where $\varepsilon$ is some small positive number. Therefore, the collection of such minimal continua is a partition of the homogeneous continuum $X$ which the group generated by the $\varepsilon$-homeomorphisms of $X$ respects. Hence Theorem 2.2 implies that the collection of such continua constitutes a continuous, terminal decomposition of $X$ into proper subcontinua. Theorem 2.3 implies, however, that each element of such a decomposition is cell-like, and hence $T$ cannot be essentially embedded.

This completes the proof of the theorem.

Since $H^{1}(X) \neq 0$ if and only if $X$ admits an essential map into $S^{1}$, we have the following useful corollary.

COROLlARY 4.8. If $X$ is a homogeneous continuum and $H^{1}(X) \neq 0$, then the conclusion of the previous theorem is valid.

COROLlarY 4.9. Let $X$ be a homogeneous continuum such that $H^{1}(X) \neq 0$. If $X$ does not contain a proper, nondegenerate, terminal subcontinuum, then the continuum $Y$ associated with $X$ can be chosen to have the following properties: 
(1) $Y$ is an aposyndetic continuum,

(2) $K$ is a connected set,

(3) $Y$ is a two-point compactification of $K$, and

(4) The group of homeomorphisms of $Y$ fixing $Y-K$ acts transitively on $K$.

ProOF. Only condition $(3)$ needs comment. Since $H^{1}(X) \neq 0, X$ admits an essential map onto $S^{1}$. Hence $X$ can be embedded in $C_{1} \times Q$, where $C_{1}$ is a simple closed geodesic in $F$. It follows that $Y-K$ is a two-point set.

Since the covering map $p \times 1$ maps $K$ into $X$, the possibility exists of proving new results about homogeneous continua by understanding aposyndetic continua such as $Y$. We begin such a program in the next section.

5. Cut points in lifts of homogeneous continua. Let $X$ be a homogeneous continuum that admits an essential map into a graph. Assume $X$ contains no proper, nondegenerate, terminal subcontinua (in other words, the decomposition of Theorem 4.7 has been done).

In this section we show that either $X$ is a solenoid or the continuum $Y$ associated with $X$ is colocally connected (this means that each point has arbitrarily small open neighborhoods whose complements are connected).

We assume the notation of the previous section. In particular, $\tilde{X}$ is a certain universal cover of $X, K$ is a component of $\tilde{X}$, and $Y$ is a compactification of $K$ with remainder a certain zero-dimensional subset of the circle at infinity.

If $j: R \rightarrow S$ is a map of the real line into the space $S$, then we say both rays of $j(R)$ are dense in $S$ if each of the sets $j((-\infty,-n])$ and $j([n, \infty))$ is dense in $S$, for any natural number $n$.

THEOREM 5.1. If $X$ is homogeneous and each component of $\tilde{X}$ is homeomorphic to the real line $R$, then $X$ is a solenoid.

PROOF. It suffices to show that every proper, nondegenerate subcontinuum of $X$ is an arc, since Hagopian [10] has shown that solenoids are the only homogeneous continua with this property.

Let $A$ be a nondegenerate subcontinuum of $X$. Let $x$ belong to $A$. Note that $\operatorname{dim} X=\operatorname{dim} \tilde{X}=1$, and that $X$ contains an arc. Thus, by [27, Theorem 7], there exists a continuous injection $j: R \rightarrow X$ of the real line into $X$ such that $j(0)=x$ and both rays of $j(R)$ are dense in $X$. Let $\tilde{j}: R \rightarrow \tilde{X}$ be a lift of $j$, and let $\tilde{x}=\tilde{j}(0)$. If $K$ is the component of $\tilde{X}$ containing $\tilde{x}$, then it follows that $\tilde{j}(R)=K$. Hence both rays of $(p \times 1)(K)$ are dense in $X$.

Let $\tilde{A}=(p \times 1)^{-1}(A)$. If $A$ is embedded inessentially in $W \times Q$, then the inclusion map of $A$ into $X$ lifts to a homeomorphism of $A$ onto a subcontinuum of some component of $\tilde{X}$, so $A$ is an arc by hypothesis. If $A$ is embedded essentially in $W \times Q$, then each component of $\tilde{A}$ is unbounded, by Theorem 3.1. In particular, the component $K^{\prime}$ of $\tilde{A}$ containing $\tilde{x}$ must contain at least one of the two rays beginning at $\tilde{x}$. It follows that $(p \times 1)\left(K^{\prime}\right)$ is dense in $X$, and hence $A=X$.

Thus each proper nondegenerate subcontinuum of $X$ is an arc, and $X$ is a solenoid. This proves the theorem.

A point $x$ of a continuum $M$ is a separating point if $M-\{x\}$ is not connected. A point $x$ of a continuum $M$ is a cut point if there exists points $y$ and $z$ such that every subcontinuum of $M$ containing both $y$ and $z$ also contains $x$. 
These definitions are not universally accepted; in particular, they do not agree with those of Whyburn [31].

THEOREM 5.2. If $Y$ contains a cut point, then $K$ is homeomorphic to the real line $R$.

PrOOF. Since $Y$ is aposyndetic, each cut point of $Y$ is a separating point [14, p. 143]. One of these points has arbitrarily small neighborhoods with boundaries consisting of two points $[\mathbf{3 1}$, p. 49]. The homogeneity of $K$ implies that each point of $K$ has this property. It is easy to see that $K$ must be a one-manifold. Hence $K$ is homeomorphic to $R$.

COROLLARY 5.3. If $Y$ contains a cut point, then $X$ is a solenoid.

THEOREM 5.4. If $Y$ does not contain a cut point, then $Y$ is colocally connected.

Proof. Since $Y$ is aposyndetic, $Y$ is semi-locally-connected [14, p. 141]. Hence the theorem follows from [32, p. 737].

We have completed the proof of the following theorem.

THEOREM 5.5. If $X$ is a homogeneous continuum, if $H^{1}(X) \neq 0$, and if $X$ contains no proper, nondegenerate, terminal subcontinuum, then either $X$ is a solenoid or the continuum $Y$ is colocally connected.

6. New proofs of old results. We illustrate the power of the maximal terminal decompositions of Theorem 4.7 by giving simple proofs of two known theorems. The first result is due to the author $[\mathbf{2 4}, \mathbf{2 5}]$.

THEOREM 6.1. If $X$ is a hereditarily indecomposable, homogeneous continuum, then $X$ is tree-like.

ProOF. Since $X$ is hereditarily indecomposable, every subcontinuum of $X$ is terminal. Hence maximal proper terminal subcontinua do not exist. Thus Theorem 4.7 implies that $X$ cannot be homogeneous unless $X$ admits no essential map into a graph.

If $\operatorname{dim} X>1$, then $X$ admits an essential map onto $S^{1}$, by a result of Krasinkiewicz [15]. If $\operatorname{dim} X=1$ and $X$ is not tree-like, then $X$ admits an essential map into a graph, by a result of Case and Chamberlain [4]. Therefore, $X$ is tree-like, and the proof of the theorem is complete.

Bing [2] has proved that the pseudo-arc is homogeneous. The pseudo-arc is hereditarily indecomposable and cell-like. Hence the requirement in Theorem 4.8 that $H^{1}(X) \neq 0$ cannot be deleted.

The second theorem has been proved through a combination of results of Jones [13], Hagopian [8, 9], and the author [24].

THEOREM 1.2. If $X$ is a homogeneous, separating plane continuum, then $X$ is decomposable.

ProOF. We begin the proof in a classical fashion due to Jones [13]. Assume $X$ is indecomposable. It is well known that $X$ is atriodic and hereditarily unicoherent. By the previous theorem, $X$ contains a decomposable subcontinuum $H$. Sorgenfrey has shown that $H$ is irreducible between two points; call them $x_{0}$ and $x_{3}$. 
The continuum $H$ does not contain an indecomposable subcontinuum with interior (rel $H$ ) because of the atriodicity of $M$. Hence it follows from a classical theorem of Kuratowski that $H$ becomes an arc under a unique, minimal, monotone decomposition.

Let $k: H \rightarrow[0,3]$ be the quotient map of this decomposition, and let $H_{t}=k^{-1}(t)$. Let $x_{1}$ belong to $H_{1}$ and $x_{2}$ belong to $H_{2}$. Using the Effros property, one proves that each continuum $H_{t}, 1 \leq t \leq 2$, is a terminal subcontinuum of $H$. The atriodicity of $X$ then implies that each continuum $H_{t}, 1 \leq t \leq 2$, is a terminal subcontinuum of $X$.

Everything up to this point has been known to Jones [13, p. 48] and Hagopian [7, p. 38]. The thorny problem remaining is to extend this decomposition of $H$ to a monotone decomposition of $X$. The quotient space of this extended decomposition should be a homogeneous, planar continuum that contains an arc. Hence the quotient space would be $S^{1}$ by a result of Bing [3], and we would have obtained the contradiction that $X$ is decomposable.

The difficulty in extending this decomposition is the following: If a similar construction is carried out for a decomposable continuum $\hat{H}$ containing $x_{1}$ and $\hat{x}_{2}$, then we have two candidates for the decomposition element to contain $x_{1}$ (call them $H_{1}$ and $\left.\hat{H}_{1}\right)$. Which shall we use? Not only is it not clear that $H_{1}=\hat{H}_{1}$, it even seems plausible that $\hat{H}_{1}$ might contain all of the continuum $H$.

Hagopian [9] has recently overcome this difficulty, but the proof is not simple.

With Theorem 3.8 at our disposal, the difficulty is easy to overcome. The decomposition elements are, of course, the maximal, proper, terminal subcontinua of $X$. Moreover, if we choose $x_{0}$ and $x_{3}$ to belong to the same composant but different decomposition elements and proceed with the above construction, then we obtain the collection of terminal subcontinua $H_{t}, 1 \leq t \leq 2$. It is clear that each $H_{t}, 1 \leq t \leq 2$, is a maximal proper terminal subcontinuum of $X$, since any larger terminal subcontinuum must contain all of $H$, which contradicts the choice of $x_{0}$ and $x_{3}$.

The one point left to consider is to make sure that the irreducible continuum $H$ between $x_{0}$ and $x_{3}$ is decomposable. Maćkowiak and Tymchatyn [19, p. 31] have shown that an indecomposable subcontinuum of an atriodic homogeneous continuum is terminal. Thus if $x_{0}$ and $x_{3}$ belong to different decomposition elements, then $H$ must be decomposable.

Consequently, the quotient space is a homogeneous, plane continuum that contains an arc. Hence the quotient space is $S^{1}$, and $X$ is decomposable. This completes the proof.

\section{BIBLIOGRAPHY}

1. D. Bellamy and L. Lum, The cyclic connectivity of homogeneous arcwise connected continua, Trans. Amer. Math. Soc. 266 (1981), 389-396.

2. R. H. Bing, A homogeneous indecomposable plane continuum, Duke Math. J. 15 (1948), 729 742 .

3. _ A simple closed curve is the only homogeneous bounded plane continuum that contains an arc, Canad. J. Math. 12 (1960), 209230.

4. J. H. Case and R. E. Chamberlain, Characterizations of tree-like continua, Pacific J. Math. 10 (1960), 73-84.

5. E. G. Effros, Transformation groups and $C^{*}$-algebras, Ann. of Math. (2) 81 (1965), 3855. 
6. J. Grispolakis and E. D. Tymchatyn, On confluent mappings and essential mappings -A survey, Rocky Mountain J. Math. 11 (1981), 131-153.

7. C. L. Hagopian, Homogeneous plane continua, Houston J. Math. 1 (1975), 35-41.

8. Indecomposable homogeneous plane continua are hereditarily indecomposable, Trans. Amer. Math. Soc. 224 (1976), 339-350.

9. __ Atriodic homogeneous continua, Pacific J. Math. 113 (1984), 333-347.

10. __ A characterization of solenoids, Pacific J. Math. 68 (1977), 425-435.

11. F. Burton Jones, The aposyndetic decomposition of homogeneous continua, Topology Proc. 8 (1983), 51-54.

12. __ On a certain type of homogeneous plane continuum, Proc. Amer. Math. Soc. 6 (1955), 735-740.

13. __ Homogeneous plane continua, Proc. Auburn Topology Conf., Auburn Univ., Auburn, Ala., 1969, pp. 46-56.

14. __ Concerning aposyndetic and non-aposyndetic continua, Bull. Amer. Math. Soc. 58 (1952), 137-151.

15. J. Krasinkiewicz, Mapping properties of hereditarily indecomposable continua, Houston J. Math. 8 (1982), 507-516.

16. __ On one-point union of two circles, Houston J. Math. 2 (1976), 91-95.

17. Wayne Lewis, The pseudo-arc of pseudo-arcs is unique, Houston J. Math. 10 (1984), 227-234.

18. __ Continuous curves of pseudo-arcs, Houston J. Math. 11 (1985), 91-99.

19. T. Maćkowiak and E. D. Tymchatyn, Continuous mappings on continua II, Dissertationes Math. 225 (1984).

20. D. R. McMillan, Acyclicity in 3-manifolds, Bull. Amer. Math. Soc. 76 (1970), 942-964.

21. P. Minc and J. T. Rogers, Jr., Some new examples of homogeneous curves, Topology Proc. 10 (1985), 347-356.

22. J. T. Rogers, Jr., Solenoids of pseudo-arcs, Houston J. Math. 3 (1977), 531-537.

23. __ Decompositions of homogeneous continua, Pacific J. Math. 99 (1982), 137-144.

24. __ Homogeneous separating plane continua are decomposable, Michigan J. Math. 28 (1981), 317-321.

25. __ Homogeneous hereditarily indecomposable continua are tree-like, Houston J. Math. 8 (1982), 421-428.

26. _ Cell-like decompositions of homogeneous continua, Proc. Amer. Math. Soc. 87 (1983), 375-377.

27. __ Homogeneous curves that contain arcs, Topology Appl. 21 (1985), 95-101.

28. __ Hyperbolic ends and continua, Michigan J. Math. 34 (1987), 337-347.

29. __ Homogeneous continua, Topology Proc. 8 (1983), 213-233.

30. __ Orbits of higher-dimensional hereditarily indecomposable continua, Proc. Amer. Math. Soc. 95 (1985), 483-486.

31. G. T. Whyburn, Analytic topology, Amer. Math. Soc. Colloq. Publ., vol. 28, Providence, R.I., 1942.

32. __ Semi-locally connected sets, Amer. J. Math. 61 (1939), 733-749.

Department of Mathematics, Tulane University, New Orleans, Louisiana 70118 